\title{
MÉTODOS DE COSTOS UTILIZADOS POR INSTITUCIONES HOSPITALARIAS EN CHILE: ESTUDIO DE CASO ${ }^{1}$
}

\author{
METHODS OF COSTS USED BY HOSPITAL \\ INSTITUTIONS IN CHILE: CASE STUDY
}

\author{
Cecilia Gallegos Muñoz ${ }^{2}$ y Estela Rodríguez Quezada ${ }^{3}$
}

\section{RESUMEN}

Las condiciones y la gestión económica y social han impulsado importantes cambios en la organización y gestión de las entidades de salud, en este contexto, la gestión de sus costos y gastos resulta ser en un elemento esencial en la búsqueda de eficiencias. Por lo cual, el uso de sistemas de costos apropiados puede constituir una herramienta que permita mejorar la gestión, ya que las instituciones con un buen sistema de información pueden tomar las mejores decisiones, que por un lado les permita optimizar los recursos que dispone y por otro, mejorar la calidad del servicio.

El objetivo del presente trabajo fue identificar las prácticas de medición de costos utilizadas por Centros de Salud Familiar, Hospitales, y Clínicas de Salud de la Región del Bio-Bio en Chile.

La investigación es de tipo exploratorio, descriptivo y de corte transversal. Se utilizó una muestra por conveniencia que incluye tres Centros de Salud Familiar (CESFAM), tres Hospitales Estatales y tres Clínicas privadas. Se definieron como variables independientes el tamaño de la organización y el tipo de financiamiento y como variables dependientes: Método de Valuación de Inventarios, Sistema de Acumulación de costos, Asignación de Costos Indirectos de Fabricación, Teorías económicas del costo de producción, Teorías económicas del costo para la gestión. Para la recogida de información, se aplicó una encuesta y en algunos casos fue necesario realizar una entrevista para conocer y entender en mayor detalle el sistema de costos utilizados.

Los resultados dan cuenta de que las Instituciones cuyos fondos provienen del Estado no calculan el costo por paciente ni por prestación, más bien, acumulan sus costos por centro de responsabilidad, en tanto, las Clínicas tienen un mayor control de sus costos aunque utilizan métodos tradicionales mayoritariamente.

Palabras clave: métodos de costeo, instituciones de salud, costo del servicio, región del Bío-Bío.

Recepción: 22/03/2018. Aprobación: 7/11/2018.

\section{ABSTRACT}

The conditions and economic and social management have prompted important changes in the organization and management of health entities, in this context, the management of their costs and expenses is an essential element in the search for efficiencies. Therefore, the use of appropriate cost systems can be a tool to improve management, since institutions with a good information system can make the best decisions, which on the one hand allows them to optimize the resources available and on the other, to improve the quality of service. The objective of the present study was to identify

1 Trabajo financiado por el Proyecto de Investigación Regular denominado "Estrategias y prácticas de gestión y medición de costos en Empresas Chilenas", código DIUBB 164818 2/R, Dirección General de Investigación, Desarrollo e Innovación, Universidad del Biobío, Chile.

2 Universidad del Biobío, Facultad de Ciencias Empresariales, correo: Cecilia@ubiobio.cl

3 Universidad del Biobío, Facultad de Ciencias Empresariales, correo: erodrig@ubiobio.cl 
the cost measurement practices used by Family Health Centers, Hospitals, and Health Clinics of the Bio-Bio Region in Chile. The research is exploratory, descriptive and cross-sectional. A convenience sample including three Family Health Centers (CESFAM), three State Hospitals and three private Clinics was used. The size of the organization and the type of financing and dependent variables were defined as independent variables: Inventory Valuation Method, Cost Accumulation System, Allocation of Indirect Costs of Manufacturing, Economic Theories of Cost of Production, and Economic Cost Theories for management. To collect information, a survey was applied and in some cases it was necessary to conduct an interview to know and understand the cost system used in greater detail. The results show that the Institutions whose funds come from the State do not calculate the cost per patient or benefit; rather, they accumulate their costs per center of responsibility, whereas the Clinics have a greater control of their costs although they mainly use traditional methods.

Keywords: Cost methods, health institutions, cost of service, region Bío-Bío.

\section{INTRODUCCIÓN}

Las condiciones y la gestión económica y social han impulsado importantes cambios en la organización y gestión de las entidades de salud, siendo su objetivo prioritario en la actualidad la búsqueda de la eficiencia. Estas instituciones han presentado crecimiento en la demanda de servicios, pero también esto ha implicado mayores gastos, para lograr entregar un servicio de calidad, (A.M. Santamaría, et. al., 2015). Lo anterior implica poseer sistemas de información en el área económica, que permitan conocer, no solamente cuánto se gasta, sino también cómo se invierten los recursos, con el fin de determinar la eficiencia de los distintos procesos en las instituciones hospitalarias.

En este contexto, los sistemas de costos constituyen una herramienta para mejorar la gestión, ya que las instituciones con un buen sistema de información pueden tomar las mejores decisiones, que por un lado les permita optimizar los recursos que dispone y por otro, mejorar la calidad del servicio. También existe la visión compartida de que la contabilidad costos y de gestión es un sistema de información capaz de proveer información relevante para la gestión empresarial (Chacón, 2007). En tanto, la finalidad de un sistema de costos es representar de la forma más exacta los distintos costos en que incurre la empresa, y en lo posible que estos sean distribuidos de la mejor forma a los servicios que correspondan, solo en este caso, se obtendría un resultado satisfactorio, identificando aquellos más rentables y aquellos más consumidores de recursos.

En tanto, pese a lo importante que es contar con un sistema de información que permita la implementación de un sistema de costos en instituciones hospitalarias, no se posee conocimiento de cuáles son las prácticas utilizadas para medirlos. Los estudios realizados revelan que, "Los hospitales públicos carecen de sistemas de costos que permitan suministrar la información necesaria para la toma de decisiones gerenciales, no obstante se están dando pasos decisivos para la adecuación e implementación de los mismos" (Vargas y Gamboa, 2004:2). Lo anterior concuerda con lo señalado por Backer y Jacobsen (1970) quienes habían declarado que los sistemas de contabilidad de costos más elaborados se encontraban en compañías manufactureras.

Ahora bien, al implementar un sistema de costos entonces, se deben tener en cuenta los diferentes métodos y prácticas de costeo existentes en la literatura, tanto tradicionales como contemporáneas y elegir aquellas más se adecuen a las necesidades de información de cada empresa, además, el cálculo de los costos es fundamental para reflejar en cifras, el proceso productivo, por lo mismo es una actividad de carácter permanente (Vargas, 2005). Hay que considerar que en los últimos años se han desarrollado una serie de nuevas técnicas de gestión empresarial, que les permite a las organizaciones actuar en un mundo globalizado y cada vez más competitivo, (Figura 1). 
Figura 1: Criterios básicos en un sistema de costos

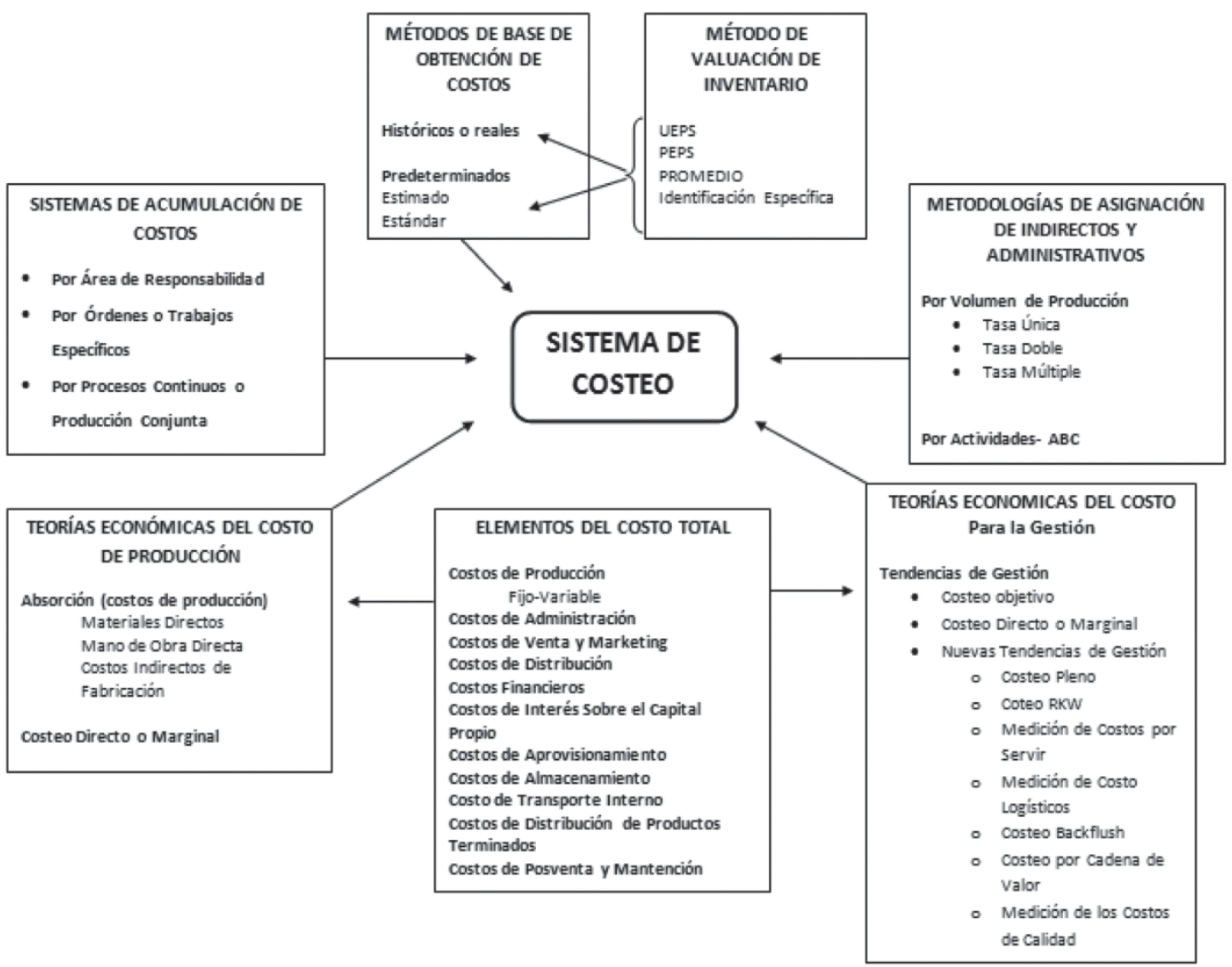

Fuente: Elaboración propia adaptada de Aguirre (2004).

En este sentido, para adoptar un método tradicional hay que considerar: el sistema de acumulación de costos, por tanto, elegir entre el sistema por órdenes de fabricación, por procesos o por centro de responsabilidad. En este contexto, Morillo (2002) señala que en los costos por órdenes se acumulan los costos de manufactura de acuerdo a las especificaciones que realice quien solicita dicho bien o servicio, en otras palabras, los costos se van acumulando en cada orden de trabajo realizada por el cliente. En tanto, en el sistema de costeo por procesos, de acuerdo a lo señalado por López-Mejía et al. (2011)is an important issue in a highly competitive field such as global markets. Some companies seeking to improve their efficiency have adopted the system Activity Based Cost (ABC se acumulan los costos de una operación o departamento en particular por todo un período, es decir, las entidades que utilizan este tipo de sistema, dividen su proceso productivo en diversas etapas o departamentos. Ahora bien, otra técnica de acu- mulación de costos es el costeo por centros de responsabilidad. En este sentido, Welsch, Hilton, Gordon y Rivera (2005) señalan que "un área o centro de responsabilidad es una unidad (o subunidad) organizacional que es dirigida por un gerente con autoridad y responsabilidades especificadas" (p.30).

En cuanto a las teorías económicas de costeo, la literatura señala que son el costeo absorbente y costeo variable. El costeo absorbente, costeo total o tradicional, es un sistema que incorpora al costo del producto, tanto los costos fijos como variables de producción, con el propósito de costear sus inventarios (Pineda, 2008), en cambio el costeo variable considera como costos del producto, solo a los costos variables.

En tanto, respecto a los métodos de base para la obtención de los costos, estos pueden ser clasificados en costos históricos o predeterminados. En el sistema de costeo histórico se utilizan costos reales, Morillo (2002), mientras que el sistema de costos predeterminados, según Neuner 
(1996) es una técnica que permite calcular los costos de manera anticipada a la culminación de la producción, con la finalidad de realizar una comparativa con los costos reales incurridos.

Por último, en cuanto a los métodos de asignación de costos indirectos, estos tienen como objetivo que los productos absorban aquellos costos indirectos que les corresponden, y entre estos se encuentran tres; tasa única, tasa doble o multitasa y costeo basado en actividades.

Ahora bien, en cuanto a las teorías económicas del costo para la gestión, son herramientas de medición y control de los costos cuyo propósito es apoyar a las organizaciones a obtener ventajas competitivas. Por lo cual, una institución de salud como cualquier tipo de organización, también necesita un sistema de costos que apoye a la gestión y no así un sistema contable tal como lo plantea Rivas (2002). En este contexto, Cokins (1992) plantea que las instituciones de asistencia médica son industrias en las que se emplea gran cantidad de mano de obra y donde las actividades de trabajo representan la mayor parte de sus costos. Del mismo modo, existe una necesidad de conocer qué servicios costear y qué actividades estarían siendo las más eficientes. Para dar solución a esta interrogante, plantea que el $A B C$ es vital importancia para el bienestar de las organizaciones de asistencia médica, tal como lo reafirma Suthummanon et. al. (2005) quien concluye que el $A B C$ es usado en numerosas instituciones de asistencia médica en el mundo.

En algunas de estas organizaciones se ha concluido que el $\mathrm{ABC}$ ayuda a fijar precios más exactos al costeo logrando así mejores prácticas, también permite identificar recursos subutilizados y reducir gastos, logrando una planificación más eficaz que si se utilizara un sistema de costeo tradicional, permitiendo a administradores de asistencia médica identificar procedimientos costosos y no rentables para la organización.

Ahora bien, en cuanto a las metodologías de costos existentes, García (2006) señala que el modelo Costes por Integración Sucesiva (CIS) "puede ser implantado como una herramienta de gestión que va a permitir, a cualquier centro sanitario, medir la eficiencia a través del proceso analítico de cálculo de costes, reflejando el beneficio o la pérdida en cada uno de los procesos, tanto en procesos hospitalizados como en ambulantes, y les va a suministrar todos los datos relevantes para poder tomar múltiples decisiones sobre su organización sanitaria".

En tanto, Montico y Velarde (2003) proponen una metodología que parte de una clasificación en dos grandes tipos de costos: variables y fijos; es decir, por un lado el sistema deberá calcular los costos variables por paciente que serán todos aquellos gastos que se originan por la atención al paciente, por ejemplo, medicamentos, material de uso médico, análisis, honorarios específicamente para ese paciente, etc. y por otro lado, se deben calcular los costos fijos o costos por utilización de los servicios, como pueden ser el costo del día/cama en cada tipo de sala (convencional, intermedio, intensivo, etc.), el costo de utilización del box quirúrgico, el costo de la consulta por especialidad, etc. no existiendo una correlación exacta entre el sistema de costos propuesto y un sistema específico de costos ya existente, sino que éste sistema es una combinación de metodologías: costos por órdenes de producción, costos por procesos y costeo basado en las actividades.

Por otro lado, Duque et al (2009) realizaron un estudio cuyo objetivo central fue analizar los sistemas de costos utilizados por las entidades prestadoras de servicios de salud en Colombia, tanto públicas como privadas donde señalan que cada vez toma más fuerza en este sector la utilización de metodologías tales como: ABC, Costeo por Protocolos, Costos por procesos, Costo por paciente/patología, Grupos Relacionados de Diagnóstico - GRD, concluyendo que se pudo comprobar el desconocimiento y disparidad de criterios aplicados por las entidades de salud en lo que tiene que ver con la información de costos y si esta situación se encuentra en entidades de gran tamaño, en las pequeñas y medianas, es escaso encontrar que apliquen de manera habitual y adecuada una metodología de costos.

Ahora bien, respecto a la metodología que se deben aplicar en el sector, son múltiples los métodos aplicables, sin embargo, se muestra una clara preferencia por metodologías tales como el Costeo Basado en Actividades o el costeo híbrido (combinación de metodologías). Los GRD en tanto, "son un sistema de clasificación de pacientes que permite medir la mezcla de casos de un hospital. Es un sistema que ha sido diseñado para poder aplicar a los hospitales formas organizativas menos jerárquicas, más horizontales, con reemplazo de las funciones tradicionales por los nuevos procesos que posibilitan las actuales tecnologías microelectrónicas, informáticas y de telecomunicaciones", (Dueñas:2003), por lo tanto, el propósito de esta investigación es identificar las prácticas de medición de costos utilizadas por las Instituciones del área de Salud, como Centros de Salud Familiar, Hospitales, y Clínicas 
de la Región del Bío-Bío en Chile, a través de un estudio de caso.

\section{METODOLOGÍA, MATERIALES Y MÉTODOS}

La investigación es de tipo exploratorio, descriptivo y de corte transversal. Se utilizó una muestra por conveniencia que incluye tres Centros de Salud Familiar (CESFAM), tres Hospitales Estatales y tres Clínicas privadas. Se definieron como variables, el tamaño de la organización y el tipo de financiamiento. Para la primera se clasificaron las Instituciones según los servicios prestados, es decir, atención primaria, (pequeña empresa) y atención secundaria (gran empresa), y para la segunda variable se consideró la fuente de donde provienen los ingresos, así tenemos financiamiento público, implica que recibe aportes estatales; financiamiento mixto, recibe financiamiento del Estado y además genera ingresos propios, y por último financiamiento privado, en donde sus ingresos provienen de pagos realizados por sus usuarios.

La información a recabar de cada una de las instituciones fueron: el método de valuación de inventarios, el sistema de acumulación de costos, el método de asignación de costos indirectos de fabricación, las teorías económicas del costo de producción y teorías económicas del costo para la gestión utilizadas por cada una de las instituciones objeto de estudio.

Para la recogida de información, se aplicó una encuesta y en algunos casos fue necesario realizar una entrevista para conocer y entender en mayor detalle el sistema de costos utilizados.

Para el análisis de la información, se identificó cada uno de los métodos utilizados por las instituciones estudiadas, y se agruparon por tipo de financiamiento y tamaño de la organización.

\section{RESULTADOS}

El estudio de caso fue realizado con un total de nueve instituciones de salud, tres Centro de Salud Familiar, tres Hospitales Estatales y tres Clínicas Privadas. La tablas a continuación muestran el resumen de la caracterización de las instituciones que formaron parte del estudio, (Ver Tabla 1, 2 y 4).

Tabla 1: Caracterización de los CESFAM

\begin{tabular}{|l|l|l|l|}
\hline Ítems & Cesfam 1 & Cesfam 2 & Cesfam 3 \\
\hline Tipo de Financiamiento & Público & Público & Público \\
Giro o actividad económica & Servicio de salud & Servicio de salud & Servicio de salud \\
$\mathrm{N}^{\circ}$ de pacientes inscritos & 16.040 & 27.796 & 11.653 \\
$\mathrm{~N}^{\circ}$ de trabajadores & 122 & 140 & 120 \\
Cargo de la persona encuestada & Jefe de Finanzas & Jefe de Finanzas & Jefe de Finanzas \\
Antigüedad en la empresa (encuestado) & 8 años & 8 años & 20 años \\
$\begin{array}{l}\text { Dpto. encuestado o área donde se } \\
\text { determinan los costos }\end{array}$ & Finanzas & Finanzas & Finanzas \\
Tipo de Atención & Primaria & Primaria & Primaria \\
¿Determinan los costos? & No & No & No \\
\hline
\end{tabular}

Fuente: Elaboración propia a partir de los resultados obtenidos en las encuestas.

Como se puede observar en la tabla 1 y dado que el tipo de servicios prestados corresponde a programas de atención primaria, los tres CESFAM son considerados pequeñas empresas, independientemente de la cantidad de usuarios atendidos. Respecto al financiamiento, los tres CESFAM, por ser instituciones estatales, son to- dos financiados por el Estado.

En cuanto a la determinación de los costos, estas instituciones no calculan el costo de sus prestaciones, solo utilizan Contabilidad Gubernamental. La Municipalidad de la jurisdicción correspondiente a la localidad del CESFAM, con la información del presupuesto anual aprobado, 
realiza la distribución del mismo, por el número de inscritos a las diferentes entidades de salud dependientes de cada municipio. (Centro de Sa- lud Familiar, Centros Comunitarios de Salud Familiar, Postas Salud Rurales y Servicio de Atención Primaria de Urgencia).

Tabla 2: Caracterización de los Hospitales.

\begin{tabular}{|c|c|c|c|}
\hline & HOSPITAL 1 & HOSPITAL 2 & HOSPITAL 3 \\
\hline Tipo de Financiamiento & Mixto & Mixto & Mixto \\
\hline $\begin{array}{l}\text { Giro o actividad } \\
\text { económica }\end{array}$ & Servicios hospitalarios & Servicios hospitalarios & Servicios hospitalarios \\
\hline $\begin{array}{l}\mathrm{N}^{\circ} \text { de pacientes o usua- } \\
\text { rios atendidos }\end{array}$ & 1.865 .104 & 518.264 & 32.000 \\
\hline $\begin{array}{l}\text { Número de } \\
\text { trabajadores }\end{array}$ & 2.065 & 600 & 145 \\
\hline $\begin{array}{l}\text { Cargo de la persona } \\
\text { encuestada }\end{array}$ & Jefe de Finanzas & Jefe de Contabilidad & Director del hospital \\
\hline $\begin{array}{l}\text { Antigüedad en la } \\
\text { empresa (encuestado) }\end{array}$ & 20 años & 8 años & 2 años \\
\hline $\begin{array}{l}\text { Dpto. encuestado o } \\
\text { área donde determinan } \\
\text { los costos }\end{array}$ & $\begin{array}{l}\text { Departamento de } \\
\text { Finanzas }\end{array}$ & $\begin{array}{l}\text { Departamento de } \\
\text { Contabilidad }\end{array}$ & $\begin{array}{l}\text { Departamento de } \\
\text { Contabilidad }\end{array}$ \\
\hline $\begin{array}{l}\text { Servicio de atención } \\
\text { prestado }\end{array}$ & Atención secundaria & Atención secundaria & $\begin{array}{l}\text { Atención primaria y } \\
\text { segundaria }\end{array}$ \\
\hline $\begin{array}{l}\text { ¿Determinan los } \\
\text { costos? }\end{array}$ & Sí & No & No \\
\hline
\end{tabular}

Fuente: Elaboración propia a partir de los resultados obtenidos en las encuestas.

Como se puede observar en la tabla 2 , los servicios prestados corresponden a atenciones secundarias, con excepción del hospital $\mathrm{N}^{\circ} 3$ que presta servicios de atención primaria y servicios secundarios, dado que es un hospital de baja complejidad, lo que significa que algunos servicios son derivados a hospitales de mayor complejidad gestionados en la red. Es decir, el Hospital $\mathrm{N}^{\circ} 1$ y $\mathrm{N}^{\circ} 2$ quedan clasificados en la categoría de hospital de gran tamaño y el Hospital $\mathrm{N}^{\circ} 3$ como pequeño.

Respecto al financiamiento, los hospitales poseen un financiamiento mixto, esto quiere decir que el financiamiento proviene del Estado y también de las atenciones particulares a sus usuarios.

En lo que refiere a la determinación de los costos, solo el Hospital $\mathrm{N}^{\circ} 1$ afirmó que calculaba los costos de cada prestación, que es aquel que atiende a un mayor número de usuarios y por ende el que posee mayor número de empleados. Sin embargo, para fijar los precios de venta, los tres hospitales lo hacen por los valores referenciales dados por el Fondo Nacional de Salud (FONASA), y según lo señalado por un hospital, a los usuarios particulares o de ISAPRES se les fija precio de venta referenciando el valor FONASA incrementado en un $100 \%$.

En tanto, de los tres hospitales uno utiliza un software propio, que es aquel que declara calcular costos, y los otros dos solo utilizan softwares estatales para almacenamiento de información. Ahora bien, dado que solo el Hospital $\mathrm{N}^{\circ} 1$ declaró que calculaba los costos de cada prestación, se presenta a continuación un resumen de los métodos utilizados por éste en la determinación de sus costos, (Ver Tabla 3). 
Tabla 3: Métodos de costeo del Hospital $N^{\circ} 1$.

\begin{tabular}{|l|l|}
\hline Método & Hospital 1 \\
\hline Método de Valuación de Inventarios & Último en entrar primero en salir (UEPS) \\
Método de base para obtención de costos & Histórico \\
Sistema de Acumulación de los costos & Por centro de responsabilidad \\
Asignación de Costos Indirectos de Fabricación & $\begin{array}{l}\text { Doble tasa de distribución (los variables por nú- } \\
\text { mero de servicios y los fijos por metro cuadrado) }\end{array}$ \\
Teorías económicas del costo de producción & Absorción \\
Teorías económicas del costo para la gestión & No utilizan \\
\hline
\end{tabular}

Fuente: Elaboración propia a partir de los resultados obtenidos en las encuestas.

Tal como se observa en la tabla 3, el Hospital $\mathrm{N}^{\circ} 1$ indica que utiliza sistemas de acumulación tradicionales (absorción), sin embargo, lo que hacen es acumular costos en los centros de responsabilidad y cuando es necesario prorratear costos de un centro a otro, lo hacen con doble tasa de asignación, siendo entonces los costos indirectos de un centro, los que se prorratean a otros, generalmente desde los centros intermedios y de apoyo hacia los centros finales. No hay indicios de cálculos de costos por prestaciones o por pacientes.

Tabla 4: Caracterización de las clínicas privadas.

\begin{tabular}{|c|c|c|c|}
\hline Ítems & Clínica 1 & Clínica 2 & Clínica 3 \\
\hline Tipo de financiamiento & Privado & Privado & Privado \\
\hline $\begin{array}{l}\text { Giro o actividad } \\
\text { económica }\end{array}$ & Servicios hospitalarios & Servicios hospitalarios & Servicios hospitalarios \\
\hline $\begin{array}{l}\mathrm{N}^{\circ} \text { de pacientes o } \\
\text { usuarios atendidos }\end{array}$ & 20.000 & 17.000 & Se desconoce \\
\hline $\begin{array}{l}\text { Número de } \\
\text { trabajadores }\end{array}$ & 900 & 259 & 360 \\
\hline $\begin{array}{l}\text { Cargo de la persona } \\
\text { encuestada }\end{array}$ & Gerente de Finanzas & Control interno & Jefe de tesorería \\
\hline $\begin{array}{l}\text { Antigüedad en la } \\
\text { empresa (encuestado) }\end{array}$ & 20 años & 8 años & 4 años \\
\hline $\begin{array}{l}\text { Dpto. encuestado o } \\
\text { área donde determinan } \\
\text { los costos }\end{array}$ & $\begin{array}{l}\text { Departamento de } \\
\text { Finanzas }\end{array}$ & $\begin{array}{l}\text { Departamento de } \\
\text { control interno }\end{array}$ & $\begin{array}{l}\text { Departamento de } \\
\text { Tesorería }\end{array}$ \\
\hline $\begin{array}{l}\text { Servicio de atención } \\
\text { prestado }\end{array}$ & Secundaria & Primaria y Secundaria & Primaria y Secundaria \\
\hline $\begin{array}{l}\text { ¿Determinan los } \\
\text { costos? }\end{array}$ & $\mathrm{Si}$ & Si & $\mathrm{Si}$ \\
\hline
\end{tabular}

Fuente: Elaboración propia a partir de los resultados obtenidos en las encuestas.

Respecto a los servicios prestados por las clínicas, todos corresponden a atención secundaria, por tanto son clasificadas como gran empresa. Estas instituciones no reciben aportes estatales, pues el capital está en manos de privados, por lo tanto se financian con los pagos que realizan sus usuarios. 
Tabla 5: Métodos de costeo de las clínicas.

\begin{tabular}{|c|c|c|c|}
\hline Métodos & Clínica 1 & Clínica 2 & Clínica 3 \\
\hline $\begin{array}{l}\text { Método de Valuación de } \\
\text { Inventarios }\end{array}$ & UEPS & $\begin{array}{l}\text { Precio Promedio } \\
\text { Ponderado (PPP) }\end{array}$ & UEPS \\
\hline $\begin{array}{l}\text { Método de base para } \\
\text { obtención de costos }\end{array}$ & $\begin{array}{l}\text { Histórico y Estimado } \\
\text { para presupuestos }\end{array}$ & Histórico & Estimado \\
\hline $\begin{array}{l}\text { Sistema de Acumulación } \\
\text { de los costos }\end{array}$ & $\begin{array}{l}\text { Por centros de } \\
\text { responsabilidad }\end{array}$ & $\begin{array}{l}\text { Por centros de } \\
\text { responsabilidad }\end{array}$ & $\begin{array}{l}\text { Por centros de } \\
\text { responsabilidad }\end{array}$ \\
\hline Asignación de CIF & $\mathrm{ABC}$ & $\mathrm{ABC}$ & $\mathrm{ABC}$ \\
\hline $\begin{array}{l}\text { Teorías económicas del } \\
\text { costo de producción }\end{array}$ & Absorción & Absorción & No \\
\hline $\begin{array}{l}\text { Teorías económicas del } \\
\text { costo para la gestión }\end{array}$ & No & No & Costeo Pleno \\
\hline
\end{tabular}

Fuente: Elaboración propia a partir de los resultados obtenidos en las encuestas.

Finalmente, como se aprecia en la tabla 5, todas las clínicas utilizan un sistema de costeo, solo que la Clínica $\mathrm{N}^{\circ} 3$ lo ha hecho utilizando nuevas tendencias de gestión aplicando costeo pleno, es decir, están calculando los costos por servicios completos desde que el paciente ingresa al recinto hospitalario hasta que es dado de alta, incluyendo tanto los costos de prestación del servicio como los gastos generales incurridos en la clínica. Tal como lo plantea la teoría, las tres instituciones distribuyen sus costos indirectos por medio del costeo ABC. Respecto al momento en que los calculan, dos de ellas lo hacen antes del ingreso del paciente, puesto que en todos los casos necesitan realizar estimaciones para entregar presupuestos a sus usuarios, además de lo anterior, la clínica uno y dos los calculan de forma histórica una vez que el servicio ha sido prestado. Para la valorización de inventarios dos Clínicas utilizan sistema UEPS y una lo hace con método precio promedio ponderado.

Ahora bien, al relacionar el tamaño de la organización y su financiamiento con las prácticas de medición de costos, en el caso del tamaño, no se encontró relación ya que los hospitales pese a ser instituciones grandes por el tipo de servicios prestados, solo uno de ellos realiza alguna medición de costos, sin embargo, en cuanto a la relación tipo de financiamiento y prácticas de medición de costos, se puede constatar que las instituciones privadas sí han avanzado en estas mediciones.

\section{DISCUSION, CONCLUSIONES}

La literatura plantea que los sistemas de costos constituyen una herramienta para mejorar la gestión. La contabilidad costos y de gestión es un sistema de información capaz de proveer información relevante, que permite identificar aquellos productos o servicios más costosos o menos eficientes, de tal forma de poder intervenir en aquellos, gestionando los recursos de manera más eficiente, y realizando propuestas concretas de mejoras. En este sentido es crucial para una institución de salud, contar con un sistema de información que les permita mejorar su eficiencia y reducir costos en este último caso especialmente para las instituciones públicas quien cada vez tienen que atender más patologías con escasos recursos. Considerando lo anterior, y a la luz de los resultados, los Cesfam analizados no cuentan con dicha información, por tanto existe poca posibilidad de implementar mejoras desde el punto de vista de obtención y utilización de recursos. Es curioso lo ocurrido con los Hospitales, ya que siendo instituciones de mayor tamaño tampoco cuentan con un sistema de costos que les provea información para mejorar su gestión, solo uno de ellos ha manifestado realizar cálculos de costos, y aquel que lo realiza lo hace disponiendo de un software para ello, lo que implica que es necesario invertir recursos para gestionar los costos en una organización, de los que muchas veces las instituciones públicas no disponen. 
Los resultados concuerdan con lo plateado por Vargas y Gamboa (2004); Backer y Jacobsen (1970), quienes señalan que los hospitales públicos carecen de sistemas de costos que permitan suministrar la información necesaria para la toma de decisiones gerenciales, por lo que, los avances más significativos en la materia se encuentran en empresas manufactureras.

Respecto a los métodos utilizados, el único hospital que ha avanzado en el cálculo de los costos lo hace solo con métodos tradicionales, como lo es el método por absorción, sin mostrar avances en la utilización de otros métodos más adecuados para la toma de decisiones gerenciales.

En lo que respecta a las clínicas, pese a que todas ellas plantan la utilización del método de costeo $\mathrm{ABC}$, tal como lo recomienda la literatura para este tipo de empresas, dos de ellas señalan utilizar método de acumulación por absorción, lo que da pie a entender que más que la utilización del método $\mathrm{ABC}$ lo que están realizando es la distribución de los costos indirectos de fabricación utilizando para ello, múltiples tasas de asignación y no la determinación de actividades cuyos costos sean traspasados a los objetos de costos, propiamente tal. Solo una de ellas señala la utilización del método $\mathrm{ABC}$ con la teoría del costo pleno, que sería la lógica de este método, puesto que para la determinación de las actividades y los recursos que serán traspasados a ellas, se deben considerar los costos indirectos de, en este caso los servicios, como también los gatos incurridos en la institución.

Ahora bien, a raíz de los resultados obtenidos, se puede concluir que, aquellas Instituciones financiadas por el Estado, como lo son los CESFAM y Hospitales públicos no determinan el costo de las prestaciones que realizan ni el costo por paciente, solo acumulan costos por centros de responsabilidad, dado que los ingresos provienen del Estado y con ello deben cubrir todos sus costos, sin verificar ni analizar aquellas prestaciones más costosas y si realmente el valor asignado a ellas alcanza a cubrir los requerimientos. Por lo tanto, queda de manifiesto que el aplicar o no un sistema de costos no depende del tamaño de la organización, sino del tipo de financiamiento. Asimismo, es importante destacar que en un hospital público sí han avanzado en la determinación de costos, y que esto depende más bien de la voluntad de sus directivos que de una exigencia ministerial de la misma. Seña- laron además haber intentado aplicar $\mathrm{ABC}$ pero haber fracasado en el intento, esto se debe a que se requiere capacitar a la gente, invertir en tecnología que entregue información requerida, y esto consume una gran cantidad de recursos que para las instituciones públicas es escaso.

Puesto que en instituciones hospitalarias existen muchos costos indirectos, queda de manifiesto que el sistema más adecuado para distribuirlos es el sistema $A B C$ ya que se pueden obtener costos más exactos para las prestaciones y/o por paciente, lo que ayudará en la gestión y distribución de los recursos. En cuanto a los sistemas tradicionales de costeo el más utilizado es el método por absorción, el cual tiene como virtud ser el método aceptado financieramente. Dado que en las instituciones hospitalarias existen distintas unidades que tienen que ver con la atención del paciente, es importante que los costos se acumulen por centros de responsabilidad, ya que este método permite descentralizar la información y toma de decisiones en distintas unidades y cada una de ellas bajo la tutela y supervisión de un responsable idóneo.

Las instituciones privadas a pesar que han avanzado respecto a las instituciones estatales en la determinación de sus costos, solo una de ellas lo ha hecho incursionando hacia nuevos enfoques de gestión como es la propuesta del enfoque de costeo pleno, las otras permanecen en el sistema de costeo por absorción, tal vez porque este sistema les reporta además información para la contabilidad financiera. Finalmente es importante destacar que se está velando por fijar mejores metas sanitarias, se habla de trabajar con eficiencia y eficacia, sin embargo, no se dan directrices para la implementación de sistemas de costos y sobre todo de nuevas tendencias de gestión que permitan cumplir con los objetivos a las Instituciones de Salud Pública en Chile.

Finalmente, dado los distintos grados de avance en que se encuentran las instituciones dependiendo si son privadas o públicas, existen recomendaciones distintas para cada una de ellas, en primer lugar para ambos tipos de instituciones es recomendable la determinación de costos por paciente, y/o por patología, que las instituciones del Estado determinen sus costos por los métodos Directo y distribuyan sus costos indirectos con multitasas, y que las instituciones privadas avancen en nuevos enfoques de gestión como el costeo pleno y distribuyan sus costos indirectos con el método $\mathrm{ABC}$. 


\section{REFERENCIAS BIBLIOGRÁFICAS}

Aguirre, José G. (2004). Sistema de costeo, la asignación del costo total a productos y servicios. Bogotá, Colombia: Fundación Universidad de Bogotá Jorge Tadeo Lozano.

A.M. Santamaría Benhumeaa, J.E. Herrera Villalobos, P.A. Sil Jaimes, N.H. Santamaría Benhumea, M.Á. Flores Manzur y A. del Arco Ortiz. (2015). Estructura, sistemas y análisis de costos de la atención médica hospitalaria. ElServier, 3(2), 134-140.

Backer, M. y Jacobsen, L. (1970). Contabilidad de costos: Un enfoque administrativo y de gerencia. México: McGraw-Hill.

Cokins G., Stratton A. y Helbling J. (1992). An $A B C$ Managers Primer. Montvale, USA: Institute of Management Accountants.

Chacón, G. (2007). La Contabilidad de Costos, los Sistemas de Control de Gestión y la Rentabilidad Empresarial. Actualidad Contable Faces, 10(15), 29-45.

Dueñas, G. (2003). La dirección de Hospitales por productos: nuevas metodologías de cálculo y análisis de costos. Ponencia presentada al VIII congreso del Instituto Internacional de costos, Uruguay.

Duque, María; Gómez, Luis; Osorio, Jair. (2009). Análisis de los sistemas de costos utilizados en las entidades del sector salud en Colombia y su utilidad para la toma de decisiones. Revista del Instituto Internacional de Costos, 5, 495-525.

García, B. (2006). Benchmarking en hospitales públicos a partir de la información suministrada por la contabilidad de costos. Revista de contabilidad, 9(17), 55-80

López-Mejía, M., Gómez-Martinez, A. \& MarínHernadez, S. (2011). Sistema de costos ABC en la mediana empresa industrial mexicana. Cuadernos de Contabilidad, 12(30), 23-43.

Montico, E., Velarde, M. (2003). Una propuesta de costos para las instituciones de asistencia médica colectiva: costos por pacientes y patología. Ponencia presentada al VIII congreso del Instituto Internacional de costos, Uruguay.
Morillo, M. (2002). Diseño de sistemas de costeo: Fundamentos Teóricos. Actulidad Contable Faces, 5(5), 7-22.

Neuner, J. (1996). Contabilidad de Costos. Principios y Práctica. México: UTEHA.

Pineda, E. (2008). Diseño de un sistema de costos para pymes. Panorama, 2(4), 18-35.

Rivas Rubén. (2002). Sistema de Costos de una Institución Hospitalaria; Administración en Salud; XVI.

Sakesun Suthummanon, Vincent K Omachonu \& Mehmet Akcin. (2005). Applying activity based costing to the nuclear medicine unit. Healthcare services management centre.

Vargas, Vilma; Cubillán, Raiza y Gamboa, Teresa. (2004). La eficiencia en un hospital de Maracaibo: su relación con la oferta y la demanda de servicio. Revista de Ciencias Sociales. X(2), 260-274.

Vargas, L. (2005). Contabilidad de costos 7a ed. Chile: SOELCO. 